\title{
CONDUÇÃO NERVOSA EM NERVOS DA MÃO E O FATOR FISIOLÓGICO IDADE
}

\author{
Berenice Cataldo Oliveira Valerio', João Antonio Maciel Nóbrega², \\ Charles Peter Tilbery'
}

\begin{abstract}
RESUMO - Muitos pesquisadores têm estabelecido a influência do fator fisiológico idade nos parâmetros do estudo da condução nervosa sensitiva e motora, e que estas alterações podem levar a erros diagnósticos. O objetivo deste estudo foi estabelecer as alterações dos parâmetros do estudo da condução nervosa sensitiva e motora dos nervos mediano e ulnar em relação à idade. Os dados foram coletados de 92 voluntários, na sua maioria funcionários da Santa Casa de São Paulo: 61 mulheres e 31 homens, em faixa etária dos 13 aos 74 anos, com média de 36,3 anos. Em nosso estudo foi observado que, com o envelhecimento, ocorre diminuição da velocidade de condução nervosa sensitiva e motora associada a redução da amplitude do potencial evocado.
\end{abstract}

PALAVRAS-CHAVE: condução nervosa, mão, nervos periféricos, eletromiografia.

\section{Neural conduction in hand nerves and the physiological factor of age}

ABSTRACT - Many researchers have established the influence of physiological factors as age, for the parameters of the study of the motor and sensitive conduction. The objective of this study was to analyze the influence of the variable age in the study of the motor and sensitive nervous conduction of the median and ulnar nerves. The data were collected from 92 volunteers: 61 women and 31 men. Their age was from 13 to 74 years old, with a mean of 36.3 years. Most of them were employees at Santa Casa de São Paulo. It was observed that a reduction in the velocity of sensitive and motor nervous conduction takes place with the age. This reduction is associated with a reduction in the amplitude of the evoked potential.

KEY WORDS: neural conduction, hand, peripheral nerve, electrophysiological, study, electromyography, study.

A eletroneuromiografia é o método de estudo neurofisiológico que registra a atividade elétrica gerada no sistema neuromuscular, sendo amplamente usada para identificar e localizar lesões do sistema nervoso periférico ${ }^{1-5}$. Gilliatt e Sears, em $1958^{6}$, descreveram o uso da latência sensitiva no diagnóstico de várias lesões do sistema nervoso periférico. Em 1961, Downie e Newell ${ }^{7}$ realizaram o estudo da condução nervosa sensitiva nos nervos mediano e ulnar, em um grupo de indivíduos normais, em diferentes faixas etárias, e observaram que a velocidade de condução nervosa sensitiva (VCNS) diminuía progressivamente após a idade de 35 anos, numa taxa de 0,4 metros por segundo por ano de vida. O estudo da condução nervosa aparentemente sofre influência de muitos fatores, tais como diâmetro do axônio, grau de mielinização do nervo, técnicas de mensuração e fatores fisiológicos ${ }^{3,8-12}$.

Entre os fatores fisiológicos que têm um efeito no estudo da condução nervosa sensitiva e motora, podendo levar a alterações nos parâmetros da resposta evocada, podemos citar idade, sexo, circunferência do dedo, raça, temperatura, altura e a variação entre a mão direita e esquerda ${ }^{1,3,13}$. O conhecimento destes fatores fisiológicos é importante porque eles podem levar a resultados falso-positivos e ocasionalmente falso-negativos, predispondo a erros diagnósticos.

Neste estudo, nós avaliamos a influência da variável idade na condução nervosa sensitiva e motora em um grupo de indivíduos normais, com idade variando de 13 a 74 anos.

\section{MÉTODO}

Os dados foram coletados de 92 mãos, de 92 voluntários, na sua maioria funcionários da Santa Casa de São Paulo. Todos compareceram espontaneamente ao Serviço de Eletroneuromiografia da Disciplina de Neurologia da

'Disciplina de Neurologia, Departamento de Clínica Médica da Faculdade da Santa Casa, São Paulo SP, Brasil; ${ }^{2}$ Setor de Neurofisiologia Clínica, Escola Paulista de Medicina / Universidade de São Paulo(EPM/UNIFESP), São Paulo SP, Brasil.

Recebido 7 Março 2003, recebido na forma final 22 Agosto 2003. Aceito 26 Setembro 2003.

Dra. Berenice Cataldo Oliveira Valerio - Rua Dom Raimundo de Brito 47 - 01526-050 São Paulo SP-Brasil. E-mail: charlespt@einstein.br 
Santa Casa de São Paulo, e após ter sido detalhado o procedimento assinaram o consentimento informado.

Dos 92 voluntários, 61 eram mulheres e 31 eram homens, na faixa etária dos 13 aos 74 anos com média de 36,3 anos. Foi realizado o estudo da condução nervosa sensitiva e motora nos nervos mediano e ulnar nas 92 mãos, sendo 56 à direita e 36 à esquerda. 0 estudo foi efetuado em apenas uma das mãos, sendo o dimídio direito ou esquerdo escolhido aleatoriamente, não se levando em consideração a dominância da mão. Foram excluídos do estudo os indivíduos com história ou evidência de neuropatia periférica, doenças do sistema nervoso central, diabetes mellitus, uso excessivo de álcool atual ou anterior e queixas de dor crônica ou parestesias nos membros. Ao exame neurológico, todos apresentavam força muscular e sensibilidade normal.

A temperatura da pele foi medida com termômetro digital de superfície na falange distal de todos os dígitos e, se abaixo de $32^{\circ} \mathrm{C}$, o membro a ser examinado era aquecido com o auxílio de bolsa de água quente. Durante o procedimento, a mensuração da temperatura foi realizada várias vezes $\mathrm{e}$, se necessário, o membro era reaquecido e mantido entre 32 e $35^{\circ} \mathrm{C}$.

Para o estudo da condução nervosa sensitiva e motora foi usado eletromiógrafo Nihon Koden, Neuropack, ME $5504 \mathrm{~K}$, de quatro canais. A calibração do osciloscópio foi, para a varredura no estudo sensitivo e motor, $2 \mathrm{~ms} /$ divisão. A sensibilidade foi de 2 ou $5 \mathrm{mV} /$ divisão para o estudo motor e 10, 20 ou $50 \mu \mathrm{V} /$ divisão para o estudo sensitivo, dependendo da amplitude do potencial evocado.

No estudo da condução nervosa sensitiva, foi usado registro ortodrômico do potencial de ação do nervo sensitivo (PANS), sendo o eletrodo de registro colocado sobre a prega proximal do pulso. Os eletrodo de registro eram montados sobre uma barra fixa de plástico, com uma distância fixa inter eletrodos de $2 \mathrm{~cm}$. Para o nervo mediano, ele foi colocado entre os tendões dos músculos palmar longo e flexor radial do carpo. Para o nervo ulnar, ele foi colocado justa lateral ao tendão do músculo flexor ulnar do carpo.

Tabela 1. Número de voluntários em cada subgrupo, segundo a faixa etária.

\begin{tabular}{ccc}
\hline Grupo & Faixa etária & No de indivíduos $^{\circ}$ \\
\hline I & $10-20$ anos & 11 \\
II & $21-30$ anos & 26 \\
III & $31-40$ anos & 28 \\
IV & $41-50$ anos & 8 \\
V & $51-60$ anos & 10 \\
VI & acima dos 60 anos & 9 \\
Total & & 92 \\
\hline
\end{tabular}

Os ramos digitais foram estimulados através de par de eletrodos em anel, sendo o cátodo colocado na interfalange proximal e o anôdo na interfalange distal do II, III e IV dedos para o estudo do nervo mediano e no $\checkmark$ dedo para o nervo ulnar. No I dedo, o cátodo foi colocado na junção metacarpofalangeana e o ânodo na junção interfalangeana para o estudo do ramo digital do nervo mediano.

No estudo da condução nervosa motora foi usado um par de eletrodos de superfície de disco para o registro do potencial de ação muscular composto (PAMC). Para o nervo mediano, o eletrodo de registro foi colocado na região tenar, sendo o ativo sobre o ponto motor do músculo abdutor curto do polegar e o referência na junção metacarpo falangeana. $O$ nervo foi estimulado distalmente, no pulso, entre o tendão dos músculos flexor radial do carpo e palmar longo e, proximalmente, no cotovelo, entre o tendão do músculo biceps braquial e a artéria braquial. Para o nervo ulnar, o eletrodo de registro foi colocado na região hipotenar, sendo o ativo sobre o ponto motor do músculo abdutor do $\mathrm{V}$ dedo e o referência na junção metacarpo-falangeana. $O$ nervo foi estimulado distalmente justa lateral ao tendão do músculo flexor ulnar do carpo e proximalmente no cotovelo, no sulco do ulnar.

A latência do PANS e do PAMC foi medida no início da deflexão da onda. A amplitude foi mensurada do pico negativo ao pico positivo do potencial evocado.

A amostra selecionada foi submetida a dois estudos estatísticos: estudo descritivo e estudo analítico.

\section{RESULTADOS}

Dos 92 voluntários, 31 eram homens $(34,0 \%)$ e 61 mulheres $(66,0 \%)$. As idades variaram de 13 a 74 anos com média de 36,3 e desvio padrão de 14,6. Foi observada maior concentração de indivíduos na faixa etária dos 21 aos 40 (Tabela 1).

Passaremos a descrever os valores mínimo, má-

Tabela 2. Valor mínimo, máximo, média aritmética e desvio padrão da velocidade de condução nervosa sensitiva do nervo mediano e ulnar.

\begin{tabular}{lcccc}
\hline Nervo & $\begin{array}{c}\text { Mínimo } \\
(\mathrm{m} / \mathrm{s})\end{array}$ & $\begin{array}{c}\text { Máximo } \\
(\mathrm{m} / \mathrm{s})\end{array}$ & Média & $\begin{array}{c}\text { Desvio } \\
\text { padrão }\end{array}$ \\
\hline Med- I dedo & 45 & 66 & 55,8 & 4,5 \\
Med- II dedo & 45 & 66 & 58,2 & 3,7 \\
Med-III dedo & 42 & 67 & 57,4 & 4,4 \\
Med- IV dedo & 38 & 70 & 58,1 & 6,1 \\
Med-palma- punho & 35 & 65 & 54,9 & 6,1 \\
Ulnar-IV dedo & 54 & 75 & 62,1 & 4,7 \\
Ulnar-V dedo & 52 & 73 & 59,3 & 3,7 \\
\hline Med, mediano; m/s, metros por segundo. & &
\end{tabular}


Tabela 3. Valor mínimo, máximo, média aritmética e desvio padrão da velocidade de condução nervosa motora do nervo mediano e ulnar.

\begin{tabular}{lcccc}
\hline Nervo & $\begin{array}{c}\text { Mínimo } \\
(\mathrm{m} / \mathrm{s})\end{array}$ & $\begin{array}{c}\text { Máximo } \\
(\mathrm{m} / \mathrm{s})\end{array}$ & Média & $\begin{array}{c}\text { Desvio } \\
\text { padrão }\end{array}$ \\
\hline Mediano & 51 & 65 & 58,0 & 2,9 \\
Ulnar & 51 & 68 & 59,1 & 3,4 \\
\hline
\end{tabular}

$\mathrm{m} / \mathrm{s}$, metros por segundo.

Tabela 4. Valor mínimo, máximo, média aritmética e desvio padrão da amplitude dos potenciais sensitivos do nervo mediano e ulnar.

\begin{tabular}{lcccc}
\hline Nervo & $\begin{array}{c}\text { Mínimo } \\
(\mu \mathrm{V}))\end{array}$ & $\begin{array}{c}\text { Máximo } \\
(\mu \mathrm{V})\end{array}$ & Média & $\begin{array}{c}\text { Desvio } \\
\text { padrão }\end{array}$ \\
\hline Med- I dedo & 02 & 107 & 44,1 & 19,7 \\
Med- II dedo & 05 & 56 & 28,7 & 11,8 \\
Med-III dedo & 04 & 65 & 33,9 & 14,9 \\
Med- IV dedo & 05 & 44 & 20,9 & 9,3 \\
Med-palma- punho & 17 & 262 & 113,8 & 43,7 \\
Ulnar-IV dedo & 05 & 41 & 18,2 & 7,1 \\
Ulnar-V dedo & 08 & 45 & 22,3 & 8,1 \\
\hline Med, mediano; $\mu \mathrm{V}$, micro volt. & & &
\end{tabular}

Med, mediano; $\mu \mathrm{V}$, micro volt.

Tabela 5. Valor mínimo, máximo, média aritmética e desvio padrão da amplitude dos potenciais de ação muscular composto dos nervos mediano e ulnar.

\begin{tabular}{lcccc}
\hline Nervo & $\begin{array}{c}\text { Mínimo } \\
(\mu \mathrm{V})\end{array}$ & $\begin{array}{c}\text { Máximo } \\
(\mu \mathrm{V})\end{array}$ & Média & $\begin{array}{c}\text { Desvio } \\
\text { padrão }\end{array}$ \\
\hline Mediano & 7.740 & 33.100 & $18.333,3$ & $5.264,7$ \\
Ulnar & 6.270 & 16.900 & $11.072,9$ & $2.262,3$ \\
\hline
\end{tabular}

$\mu \mathrm{V}$, microvolt. ximo e média aritmética com desvio padrão dos diferentes parâmetros do estudo da condução nervosa sensitiva e motora. Para a condução nervosa sensitiva, as amplitudes estão em $\mu \mathrm{V}$, e as velocidades em $\mathrm{m} / \mathrm{s}$. Para a condução nervosa motora as amplitudes estão em $\mu \mathrm{V}$, e as velocidades em $\mathrm{m} / \mathrm{s}$.

A medida da VCNS nos diferentes segmentos pesquisados, com seu valor mínimo, máximo e média aritmética com desvio padrão são mostradas na Tabela 2.

A medida da VCNM obtida no nervo mediano e ulnar, com registro na região tenar e hipotenar respectivamente, com seu valor mínimo, máximo e média aritmética com desvio padrão respectivo são mostradas na Tabela 3.

O valor mínimo, máximo, média e desvio padrão da amplitude do PANS obtido nos diversos segmentos do nervo mediano e ulnar são mostradas na Tabela 4.

O valor mínimo, máximo, média e desvio padrão da amplitude do PAMC obtido do nervo mediano e ulnar, com registro na região tenar e hipotenar é mostrado na Tabela 5.

No estudo comparativo dos dados obtidos nas diferentes faixas etárias, divididas por décadas, observa-se:

a. Redução significativa da amplitude do PANS, na faixa etária maior que 51 anos de idade, em todos os segmentos pesquisados (Tabela 6)

b. Redução significativa da amplitude do PAMC do nervo mediano e ulnar, na faixa etária acima dos 51 anos de idade para o nervo ulnar e acima dos 60 anos de idade para o nervo mediano (Tabela 7)

c. Diminuição significativa da velocidade de condução nervosa sensitiva no grupo maior de

Tabela 6. Média aritmética e desvio padrão (entre parênteses) da amplitude do PANS (em $\mu$ V), nas diferentes faixas etárias nos nervos mediano e ulnar (ortodrômico).

\begin{tabular}{lccccccc}
\hline Nervo & $10-20$ & $21-30$ & $31-40$ & $41-50$ & $51-60$ & $>60$ anos & $p$ \\
\hline Med-I dedo & $51,5(8,8)$ & $53,5(18,0)$ & $45,7(21,9)$ & $43,1(8,2)$ & $26,0(9,9)$ & $23,3(14,9)$ & $<0,001$ \\
Med- II dedo & $31,8(8,8)$ & $33,5(10,8)$ & $31,2(12,2)$ & $27,5(7,6)$ & $18,9(8,8)$ & $15,2(6,0)$ & $<0,001$ \\
Med-III dedo & $36,3(9,3)$ & $40,6(13,0)$ & $39,1(13,7)$ & $30,4(9,4)$ & $20,8(13,2)$ & $13,4(5,1)$ & $<0,001$ \\
Med-IV dedo & $25,3(8,4)$ & $24,7(8,9)$ & $22,0(7,4)$ & $22,0(10,1)$ & $11,4(6,3)$ & $10,4(4,9)$ & $<0,001$ \\
Med-p-p & $133,9(27,8)$ & $123,4(43,3)$ & $122,1(43,4)$ & $120,0(43,6)$ & $83,1(32,0)$ & $64,1(24,0)$ & 0,003 \\
Ulnar-IV dedo & $18,3(5,9)$ & $22,1(7,4)$ & $19,3(5,7)$ & $19,0(6,0)$ & $12,0(5,3)$ & $10,0(3,1)$ & $<0,001$ \\
Ulnar-V dedo & $22,5(5,4)$ & $26,0(8,1)$ & $23,1(8,7)$ & $22,0(6,8)$ & $17,9(5,6)$ & $14,3(4,1)$ & 0,002 \\
\hline
\end{tabular}

Med, mediano; $p$, significância; $p-p$, palma-punho; $\mu \mathrm{V}$, microvolt. 
Tabela 7. Valores mínimo, máximo, média aritmética e desvio padrão da amplitude do PAMC (em $\mu V$ ) nos nervos mediano e ulnar, nas diferentes faixas etárias.

\begin{tabular}{|c|c|c|c|c|c|c|c|c|}
\hline \multirow[t]{2}{*}{ Faixa Etária } & \multicolumn{4}{|c|}{ Nervo Mediano } & \multicolumn{4}{|c|}{ Nervo Ulnar } \\
\hline & Mínimo & Máximo & Média & DP & Mínimo & Máximo & Média & DP \\
\hline $10-20$ & 12.200 & 33.100 & $22.727,3$ & $6.115,9$ & 9.900 & 13.800 & $11.563,6$ & $1.160,4$ \\
\hline $21-20$ & 11.300 & 29.500 & $19.642,3$ & $4.484,7$ & 7.990 & 16.900 & $11.992,3$ & $1.908,0$ \\
\hline $31-40$ & 9.350 & 27.300 & $17.787,5$ & $5.141,6$ & 6.460 & 15.400 & $11.566,8$ & $2.425,4$ \\
\hline $41-50$ & 11.800 & 23.700 & $17.875,0$ & $4.897,7$ & 9.010 & 15.600 & $11.086,2$ & $2.239,1$ \\
\hline $51-60^{a}$ & 11.800 & 22.100 & $16.807,0$ & $3.822,4$ & 6.990 & 10.400 & $8.637,0$ & $1.294,9$ \\
\hline Maior $60^{b}$ & 7.740 & 19.800 & $12.982,2$ & $3.244,9$ & 6.270 & 11.100 & $8.975,5$ & $1.653,0$ \\
\hline
\end{tabular}

DP, desvio padrão. a, PAMC do nervo mediano 51-60 vs.10-20, $p=0,007$ (teste t-Student) e para o nervo ulnar $p<0,001$.

Tabela 8. Média aritmética e desvio padrão da velocidade de condução nervosa sensitiva $(\mathrm{m} / \mathrm{s})$, com seu respectivo desvio padrão, nas diferentes faixas etárias nos nervos mediano nervo ulnar.

\begin{tabular}{lccccccc}
\hline Nervo & $10-20$ & $21-30$ & $31-40$ & $41-50$ & $51-60$ & $>60$ & $p$ \\
\hline Med- I dedo & $57,1(3,5)$ & $56,8(4,2)$ & $56,1(4,5)$ & $56,2(3,8)$ & $54,3(3,9)$ & $51,4(5,2)$ & 0,026 \\
Med- II dedo & $59,3(2,2)$ & $59,1(2,8)$ & $58,7(3,4)$ & $58,6(3,2)$ & $56,2(3,4)$ & $53,5(5,0)$ & 0,004 \\
Med-III dedo & $59,4(2,1)$ & $58,8(2,9)$ & $58,1(3,5)$ & $58,1(3,2)$ & $54,9(4,7)$ & $50,9(6,4)$ & $<0,001$ \\
Med- IV dedo & $60,0(4,0)$ & $60,3(4,8)$ & $58,5(5,0)$ & $59,6(4,3)$ & $56,4(6,4)$ & $49,0(7,7)$ & $<0,001$ \\
Med-p-p & $56,2(3,0)$ & $57,1(4,3)$ & $55,6(5,3)$ & $54,7(6,6)$ & $52,1(6,3)$ & $47,4(9,2)$ & 0,006 \\
Ulnar-IV dedo & $61,3(3,4)$ & $64,1(4,9)$ & $62,4(4,3)$ & $62,3(4,9)$ & $61,3(4,0)$ & $56,6(3,1)$ & 0,002 \\
Ulnar-V dedo & $58,8(1,9)$ & $60,7(3,7)$ & $59,9(3,4)$ & $59,7(5,1)$ & $57,8(2,8)$ & $55,2(2,2)$ & 0,002 \\
\hline
\end{tabular}

Med, mediano; $p$-p, palma-punho; $p$, significância.

Tabela 9. Valores mínimo, máximo, média aritmética e desvio padrão da velocidade de condução nervosa motora $(\mathrm{m} / \mathrm{s})$ dos nervos mediano e ulnar, nas diferentes faixas etárias.

\begin{tabular}{|c|c|c|c|c|c|c|c|c|}
\hline \multirow[t]{2}{*}{ Faixa Etária } & \multicolumn{4}{|c|}{ Nervo Mediano } & \multicolumn{4}{|c|}{ Nervo Ulnar } \\
\hline & Mínimo & Máximo & Média & $\mathrm{DP}$ & Mínimo & Máximo & Média & $\mathrm{DP}$ \\
\hline $10-20$ & 55 & 60 & 57,7 & 1,7 & 55 & 68 & 60,0 & 3,4 \\
\hline $21-20$ & 54 & 65 & 59,1 & 2,6 & 55 & 67 & 60,1 & 2,9 \\
\hline $31-40$ & 52 & 64 & 57,5 & 2,5 & 52 & 65 & 58,8 & 2,8 \\
\hline $41-50$ & 57 & 65 & 60,4 & 3,6 & 56 & 65 & 60,4 & 3,3 \\
\hline $51-60$ & 52 & 61 & 57,5 & 2,5 & 51 & 66 & 58,0 & 4,8 \\
\hline Maior 60 & 51 & 62 & 55,4 & 3,6 & 52 & 61 & 56,4 & 3,0 \\
\hline
\end{tabular}

DP, desvio padrão. Significância do nervo mediano, $p=0,002$; do nervo ulnar, $p=0,045$.

60 anos em todos os segmentos pesquisados (Tabela 8)

d. Diminuição significativa da velocidade de condução nervosa motora no nervo mediano e ulnar na faixa etária acima dos 60 anos de idade (Tabela 9).

\section{DISCUSSÃO}

Os valores obtidos em nosso estudo não podem ser comparados estatisticamente aos demais valores referidos na literatura devido a diferenças na metodologia e a diferença nas faixas etárias estudadas nas pesquisas anteriores. Apesar disso, 
os dados obtidos no nosso material são concordantes aos obtidos por outros autores ${ }^{10,11,14-16}$.

Em relação à influência da variável idade no estudo da condução nervosa sensitiva e motora, podemos dizer que foi observada redução estatisticamente significante da amplitude do PANS nos nervos mediano e ulnar, em todos os segmentos pesquisados, na faixa etária acima dos 51 anos. $O$ mesmo ocorreu com a amplitude do PAMC, porém a redução foi significativa a partir dos 51 anos para o nervo ulnar e a partir dos 60 anos para o nervo mediano. Em relação a VCNS e VCNM os dados obtidos mostram diminuição estatisticamente significativa de ambas em indivíduos normais com mais de 60 anos de idade.

As diminuições da VCN e da amplitude do potencial evocado associado ao aumento da idade têm sido bem documentadas na literatura. Já em 1963, Mayer ${ }^{17}$, determinando os valores normais da condução nervosa em humanos, estudou diversos nervos nas extremidades superior e inferior. Observou que a velocidade de condução nervosa sensitiva e motora diminuía após os 50 anos de idade, sendo mais acentuada nos nervos testados nos membros superiores e mais proeminente distalmente, e que esta alteração estava associada a uma redução da amplitude do potencial evocado. No mesmo ano vários outros estudos demonstraram o mesmo achado, et al. ${ }^{18-20}$

Mais recentemente, Grandini et al., em $1992^{16}$, realizaram um estudo semelhante ao nosso, para a obtenção dos valores normais dos diferentes parâmetros do estudo da condução nervosa em um grupo de indivíduos brasileiros e saudáveis, e observaram decréscimo da amplitude do potencial evocado dos nervos sural e tibial posterior associado à diminuição da VCNS e prolongamento da latência motora terminal nos grupos de maior faixa etária.

Como o estudo da condução nervosa sensitiva e motora auxilia no diagnóstico de várias patologias, incluindo lesão de plexo braquial e neuropatias, deve-se sempre ter em mente que o envelhecimento, geralmente após os 50 anos de idade, pode levar a modificações nos parâmetros do estudo da condução nervosa, para não se concluir por um diagnóstico falso-negativo ou falso-positivo ${ }^{7,21}$.

\section{CONCLUSÃO}

Nosso estudo demonstra que o fator fisiológico idade levou a redução significativa da amplitude do PANS e do PAMC na faixa etária acima dos 51 anos e diminuição significativa da velocidade de condução nervosa sensitiva e motora nos nervos mediano e ulnar na faixa etária acima dos 60 anos.

\section{REFERÊNCIAS}

1. Dumitru D. Electrodiagnostic medicine. Ed.2. Philadelphia: Hanley \& Belfus, Jan.2002.

2. Felsenthal G. Median and ulnar muscle and sensory evoked potentials. Am J Phys Med 1978;57:167-182.

3. Kimura J. Principles and variations of nerve conduction studies, In Davis FA (ED). eletrodiagnosis in diseases of nerve and muscle. Ed.3. Philadelphia; 2001:91-117.

4. Preston DC, Shapiro BE. Approach to nerve conduction studies and electromyography. Electromyography and neuromuscular disorders: clinical electrophysiologic correlations. Boston: Butterworth-Heinemann, 1998:3-7.

5. Preston DC, Shapiro BE. Anatomy and neurophysiology. Electromyography and neuromuscular disorders: clinical electrophysiologic correlations. Boston: Butterworth-Heinemann, 1998:9-21.

6. Gilliatt RW, Sears TA. Sensory nerve action potencial with peripheral nerve lesions. J Neurol Neurosurg Psychiatry 1958;21:109-118.

7. Downie AW, Newell DJ. Sensory nerve conduction in patients with diabetes mellitus and controls. Neurology 1961;11:876-882.

8. Preston DC, Shapiro BE. Basic Nerve Condiction Sduties. Electromyography and neuromuscular disorders: clinical electrophysiologic correlations. Boston: Butterworth-Heinemann, 1998:25-44.

9. Stetson DS, Albers JW, Silverstein BA, Wolfe RA. Effects of age, sex, and anthropometric factors on nerve conduction measures. Muscle Nerve 1992;15:1095-1104.

10. Cruz MA, Barrio M, Perez Conde MC, Gutierrez AM. Electrophysiological aspects of sensory conduction velocity in healthy adults. 1 . Conduction velocity from digit to palm, from palm to wrist, and across the elbow, as a function of age. J Neurol Neurosurg Psychiatry 1978; 41:1092-1096.

11. Cruz MA, Barrio M, Perez Conde MC, Ferrer MT. Electrophysiological aspects of sensory conduction velocity in healthy adults: 2 . Ratio between the amplitude of sensory evoked potentials at the wrist on stimulating different fingers in both hands. J Neurol Neurosurg Psychiatry 1978;41:1097-1101.

12. Preston DC, Shapiro BE. Artifacts and technical factors. Electromyography and neuromuscular disorders: clinical electrophysiologic correlations. Boston: Butterworth-Heinemann, 1998:85-89.

13. Lafratta $\mathrm{CW}$, Smith $\mathrm{OH}$. A study of the relationship of motor nerve conduction velocity in the adult to age, sex, and handedness. Arch Phys Med Rehabil 1964;45:407-412.

14. Correa PM, Sosa A, Lopez Acevedo CE. Nerve conduction velocities: normal values for median and ulnar nerves. Bol Asoc Med P Rico 1986; 78:191-196.

15. Nielsen VK. Sensory and motor nerve conduction in the median nerve in normal subjects. Acta Med Scand 1973;194:435-443.

16. Grandini DL, Nobrega JA, Juliano Y. Normal values of neural conduction velocity in a group of 101 subjects. Arq Neuropsiquiatr 1992;50:50-55.

17. Mayer RF. Nerve conduction studies in man. Neurology 1963;13:1021-1030.

18. Lafratta CW, Canestrari R. A comparison of sensory and motor nerve conduction velocities as related to age. Arch Phys Med Rehabil 1966;47:286-290

19. Buchthal F, Rosenfalck A. Evoked action potencials and conduction velocity in human sensory nerves. Brain Res 1966;3:1-122.

20. Melvin JL, Harris DH, Johnson EW. Sensory and motor conduction velocities in the ulnar and median nerves. Arch Phys Med Rehabil 1966; 47:511-519.

21. Fincham RW, Van Allen MW. Sensory nerve conduction in amyotrophyic lateral sclerosis. Neurology 1964,50:50-55. 